\title{
ON INCREASE OF THE SENSITIVITY AND DIRECTIVITY OF RADIO INTERFEROMETERS
}

\author{
V. A. SANAMIAN AND H. M. TOVMASIAN \\ Biurakan Astrophysical Observatory, Armenian S.S.R.
}

To discover the possibly large number of weak discrete radio sources and to determine their positions accurately is a very important and, at the same time, a difficult task. Radio telescopes with a large resolving power and a high-sensitivity receiver are necessary for this purpose.

Most investigators concentrate on working out methods of observation that will permit the discovery of the weakest possible radio sources and, as accurately as possible, the determination of their coordinates.

Increasing the time constant of the output is one of the most effective ways of raising the sensitivity of the radio telescopes for given aerial and receiver parameters. Special attention was paid to this in the Biurakan Astrophysical Observatory. Two different methods were worked out that permit time constants of about $1 \frac{1}{2}$ to 2 hours, using photographic and resonant integration of the weak noise signals.

The basic principle of photographic integration is the use of a reflecting galvanometer as an output whose scale is replaced by an ordinary photographic plate. The light of the galvanometer oscillates under the influence of the receiver noise around an average position; as a result the photographic plate blackens. Since the noise is random, the density distribution on the plate must be gaussian with the maximum density at the center of the black strip. When a weak signal from a radio source is applied for a long time to the receiver's input, the center of the blackening must be displaced. The magnitude of the received signal is determined by that displacement.

This method of integration may be applied to the phase-switching radio interferometer only when we can rectify the negative half-periods of the interference pattern.

Two identical integrating systems were applied, in which the negative half-period reversals of the interference pattern were displaced by one-quarter periods, because in weak signals reversing exactly at zero points of the interference pattern is practically impossible. Signal integration then takes place in both arms of the output equipment. Moreover, if in the first arm the center of the blackening is displaced, let us say, to the right, then in the second it will be displaced to the left and vice versa. The magnitude of the integrated signal can be determined by comparing the density distribution curves on photographic plates from both arms.

In the case of resonance integration the output of an ordinary interference radio telescope is applied to a resonant oscillation system with natural frequency equal to that of the interference pattern. This frequency depends on the radio interferometer parameters and the declination of the observed 
source. When a weak signal from a radio source is applied together with the receiver noise to the input of the resonant system, it oscillates in agreement with the alternating signal owing to the appearance of resonance. In consequence the separate lobes of the interference pattern accumulate. The random noise at the output of the interferometer has less influence on the system.

Consequently it becomes possible to distinguish weak radio sources that would not otherwise be discovered by the same interferometer if the usual observation methods were applied.

Theoretical calculations and the results of observation show that the sensitivity of the interferometers is increased more than ten times by applying both photographic and resonant methods of integration. The value of the expected gain in sensitivity is determined by the expression $\left(\tau_{n} / \tau\right)^{1 / 2}$, where $\tau$ is the usual time constant, and $\tau_{n}$ is the integration time.

The second remarkable property of these methods is the increase of the resolving power in declination of the interferometers. The resolving power in right ascension is also increased in the particular cases when the resonant integration method is used.

The increase of directivity in declination in photographic integration depends on the fact that the signal is integrated from only those sources whose interference frequency equals the resonant frequency. Thus we can define with great accuracy the declination of the observed source by determining from a set of observations the reversal period for maximum signal integration.

The increase of directivity in declination in resonant integration comes from adding the selectivity property of the resonant system to the directivity of the radio telescope. With the resonant system's increase of $Q$, the strip of influential source declinations narrows.

The increase of directivity in declination from both methods is greater as radio sources at higher declinations are observed, and where the variation of the interference lobes with declination is larger. For example, the angle between half-power points on the interferometer's "effective" directivity diagram becomes equal to about 30 minutes of arc independently of the diagram of each aerial if the resonant integration method is used; a source at declination $=60$ degrees is observed with an aerial spacing equal to 100 wavelengths; and the $Q$ of the applied resonant system is equal to about 100 .

Thus, the resolving power of the interferometer is improved by using the methods of integration. This is especially important in observations at meter wavelengths where directivity is limited by aerial dimensions.

Detailed theoretical analyses of the suggested methods, practical circuits, and results of experimental tests are quoted in articles published by the authors $[1,2,3,4]$.

\section{REFERENCES}

[1] Sanamian, V. A. Commun. Biurakan Obs. XXII, 1957.

[2] Tovmasian, H. M. Proc. Acad. Sci. Armen. S.S.R. 25, 107, 1957.

[3] Tovmasian, H. M. Proc. Acad. Sci. Armen. S.S.R. 26, 11, 1958.

[4] Tovmasian, H. M., and Burunsuzian, E. S. Commun. Biurakan Obs. XXV, 1958. 